\title{
Electrochemically active materials and wearable biosensors for the in situ analysis of body fluids for human healthcare
}

\author{
Heun Park', Woohyun Park $\mathbb{D}^{2}$ and Chi Hwan Lee (i] ${ }^{1,2,3}$
}

\begin{abstract}
The increasing demand for minimal to noninvasive in situ analysis of body fluids, such as sweat, interstitial fluid, and tears, has driven rapid development of electrochemically active materials and wearable biosensors. The mechanically soft and deformable nature of these biosensors enables them to efficiently adapt to the geometric nonlinearity of a specific part of the human body. The integration of these biosensors with a fully miniaturized wireless telemetry system enables displaying real-time data in a mobile device and/or reporting to an encrypted server for post analysis. These features are essential for the long-term, unobtrusive monitoring of biochemical activity in ambulatory care settings for improved management of many chronic diseases, such as diabetes, gout, and Parkinson's disease. Herein, we present the latest innovations of wearable electrochemical sensors tailored for human skin or eyes with a focus on their materials, designs, sensing mechanisms, and clinical implications.
\end{abstract}

\section{Introduction}

Stretchable and wearable (i.e., on-body) sensors have attracted tremendous attention for a variety of health monitoring applications due to their capabilities to interface with a specific body part in an unobtrusive manner, thus enable the long-term reliable recording of clinically useful biosignals ${ }^{1-4}$. These sensors aim to capture the physiological conditions in a human body associated with a specific disease as a result of biochemical or/ and biophysical changes with high accuracy, ${ }^{5,6}$. Wireless power and data connection to these sensors using a fully miniaturized, flexible module enable real-time data communications with smartwatches, phones, and tablets for displaying, post analyzing, and reporting to an encrypted server for the development of telehealthcare protocols ${ }^{7,8}$.

\footnotetext{
Correspondence: Chi Hwan Lee (lee2270@purdue.edu)

${ }^{1}$ Weldon School of Biomedical Engineering, Purdue University, West Lafayette, IN 47907, USA

${ }^{2}$ School of Mechanical Engineering, Purdue University, West Lafayette, IN 47907, USA

Full list of author information is available at the end of the article

These authors contributed equally: Heun Park, Woohyun Park
}

Of these sensors, wearable electrochemical sensors that enable the minimal to noninvasive monitoring of body fluids such as sweat, interstitial fluid (ISF), and tears are of particular interest as easy-to-use and portable alternatives to conventional sophisticated analytical instruments in the healthcare industry ${ }^{8,9}$. These body fluids are a rich source of important biomarkers, including ions (e.g., $\mathrm{Na}^{+}$, $\mathrm{K}^{+}, \mathrm{Ca}^{+}$, and $\mathrm{Cl}^{-}$), small molecules (e.g., glucose, lactate, uric acid, peptides, and ammonia), and proteins, thereby providing clinically useful information for the management of many metabolic diseases, such as diabetes, gout, and Parkinson's disease ${ }^{10-12}$. For instance, sweat is a representative body fluid in which the time-dependent detection of its biomarkers across different locations on the body (e.g., abdomen, forearm, and forehead) provides important insights into the metabolic status (e.g., glucose and alcohol levels $)^{10,13,14}$. ISF-another rich source of biomarkers-comprises $15-25 \%$ of the total body weight and is formed by the transcapillary exchange during blood flow $^{15}$. The biomarkers in ISF provide more systemic information than that of biomarkers in sweat due to their origins in the blood and their high sensitivity to changes

\section{(c) The Author(s) 2021}

(c) (i) Open Access This article is licensed under a Creative Commons Attribution 4.0 International License, which permits use, sharing, adaptation, distribution and reproduction cc) in any medium or format, as long as you give appropriate credit to the original author(s) and the source, provide a link to the Creative Commons license, and indicate if changes were made. The images or other third party material in this article are included in the article's Creative Commons license, unless indicated otherwise in a credit line to the material. If material is not included in the article's Creative Commons license and your intended use is not permitted by statutory regulation or exceeds the permitted use, you will need to obtain permission directly from the copyright holder. To view a copy of this license, visit http://creativecommons.org/licenses/by/4.0/. 
in local tissues ${ }^{15-17}$. In addition, the extraction of ISF is less invasive than the extraction of blood through the use of microneedles that can pierce the stratum corneum of the skin without damaging the underlying blood vessels to minimize the risk of bacterial infection ${ }^{18-20}$. Tears also have a close relationship with blood because of the continuing leakage of plasma from blood to tears through the blood-tear barrier ${ }^{21}$. A tear film $\sim 3 \mu \mathrm{m}$ thick (corresponding to $3-10 \mu \mathrm{l}$ of tear fluid) is continuously renewed during the complex interplay between the lacrimal and accessory lacrimal glands as well as the goblet cells of the conjunctiva $^{22-25}$. The biomarkers of tears can be simultaneously sampled and analyzed at the corneal surface or, more ideally, in the pocket of the inferior conjunctival fornix (near the meibomian glands) using a contact lensbased or capsule-based sensor platform ${ }^{26}$. The biomarkers of tears are also analyzed to obtain important insights into biochemical activity associated with ocular diseases such as dry eye syndrome ${ }^{27,28}$. While access to sweat is the simplest and most common among these body fluids, the volume and analyte concentration of both ISF and tears are relatively well maintained during electrochemical measurements, offering better stability and reliability in sensor dynamics ${ }^{29}$.

Several different types of wearable electrochemical sensors with tailored mechanical, electrical, and chemical properties have emerged that can establish a highly intimate interface with a specific body part, therefore enabling the extraction and detection of body fluids with high reliability. These features are realized through the use of mechanically flexible and electrochemically active nanomaterials (e.g., functionalized graphene and/or nanowires) that are capable of capturing changes in their electrical properties with respect to a biological response during on-body movements ${ }^{30-39}$. The electroanalytical detection by these sensors occurs when a target analyte is combined with the functionalized nanomaterials to either accept or donate electrons from/to electrodes. Various methods for electroanalytical detection, including potentiometry, amperometry, conductometry, and voltammetry, are currently used in which the name of each method reflects the measured electric property or its units ${ }^{40}$. For instance, potentiometry measures the electric potential (or voltage) across electrodes with a constant electric current. Amperometry measures the electric current (amperes) across electrodes with a constant electric potential. Conductometry measures the conductance of a solution (i.e., the ability of a solution to carry electric current) across electrodes with a constant alternating current (AC) potential. Voltammetry measures the charge-carrying capacity of electrodes at different voltage levels while monitoring the current. These methods also offer the advantage of being able to process the data from multiple sensors at the same time using an electrical control circuit.

Herein, we review the latest wearable electrochemical sensors tailored for the monitoring of the abovementioned three representative body fluids (sweat, ISF, and tears) in terms of their device platform, detection method, active material, target biomarker, and related human disease, as also summarized in Table 1. As illustrated in Fig. 1, we classify the sensors into three groups according to the target body fluid. In each section, we discuss (1) the effective methods to extract body fluids, (2) the basic materials and components, wireless connection configurations, and sensing mechanisms of the sensors, and (3) their implications for human health. In the conclusion section, we discuss the remaining challenges and future opportunities to further expand the applicability of these wearable electrochemical sensors for their use in a wide range of clinical practices.

\section{Wearable sweat sensors}

Wearable (i.e., on-skin) sweat sensors provide great potential to enable the continuous assessment of health physiology by analyzing sweat biomarkers in a noninvasive and unobtrusive manner ${ }^{13,14}$. In this field, the largest continuing challenge is that these sensors must remain in contact with the curvilinear surface of the skin over a prolonged period during on-body movements for the efficient collection of sweat and the in situ analysis of biomarkers. In addition, the collection procedure is tedious due to the low secretion volume and fast evaporation of sweat, and the collection time varies depending on environmental conditions (e.g., ambient temperature). Thus, these sensors typically demand higher sensitivity and selectivity than conventional analytical instruments used for blood samples, especially considering the substantially lower concentration of biomarkers (e.g., glucose) in sweat than in blood by a couple of orders of magnitude ${ }^{41}$. In the following sections, we introduce recent works on wearable sweat sensors categorized by their sweat induction and collection methods. These methods involve the use of (1) iontophoresis to induce sweating in a controlled manner and (2) microfluidics to guide and collect sweat to a reservoir via controlled capillary action. In the following section, a unique theranostic device is also discussed in which both an electrochemical sensor and a drug delivery unit are integrated into a single wearable platform, enabling controlled feedback release of therapeutic drugs in response to the sensing results. These sensors are typically fabricated via costeffective printing techniques by employing low-modulus materials such as thermoplastics, fabrics, and elastomers ${ }^{37}$. As a consequence, they are potentially disposable and do not need to be reused with different patients. 
Table 1 Overview of representative biofluids in terms of their sensing methods, sensor platforms, active materials, analytes, and diseases.

\begin{tabular}{|c|c|c|c|c|c|c|}
\hline Target body fluid & Device platform & Detection method & Active material & Target biomarker & Related human disease & Ref. \\
\hline \multirow[t]{6}{*}{ Sweat } & \multirow[t]{3}{*}{ Iontophoresis } & Amperometry & Prussian blue, AOx & Alcohol & Alcoholic hepatitis & 47 \\
\hline & & Potentiometry & Sodium ionophore & Sodium & Hyponatremia & 44 \\
\hline & & Potentiometry & Ammonia ionophore & Ammonium & Hepatitis, cirrhosis & 45 \\
\hline & \multirow[t]{2}{*}{ Microfluidic } & Voltammetry & Graphene & $\begin{array}{l}\text { Uric acid, } \\
\text { tyrosine }\end{array}$ & Gout, tyrosinemia & 56 \\
\hline & & Amperometry & GOx, LOx & $\begin{array}{l}\text { Glucose, } \\
\text { lactate }\end{array}$ & Diabetes, Ischemia & 88 \\
\hline & Theranostic & Potentiometry & GOx & Glucose & Diabetes & 58,59 \\
\hline \multirow[t]{3}{*}{ ISF } & \multirow[t]{2}{*}{ Microneedle } & Amperometry & Catechol & Melanoma & Skin cancer & 89 \\
\hline & & $\begin{array}{l}\text { Amperometry, } \\
\text { voltammetry }\end{array}$ & $\begin{array}{l}\text { Carbon paste, } \\
\text { tyrosinase }\end{array}$ & Levodopa & Parkinson's disease & 67 \\
\hline & $\begin{array}{l}\text { Reverse } \\
\text { iontophoresis }\end{array}$ & Amperometry & GOx & Glucose & Diabetes & 46 \\
\hline \multirow[t]{4}{*}{ Tears } & \multirow[t]{3}{*}{ Contact lens } & Conductometry & Chitosan-PVA, GOx & Glucose & Diabetes & 76,90 \\
\hline & & Amperometry & LOx & Lactate & Ischemia & 74 \\
\hline & & Conductometry & C-Mab & Cortisol & Stress & 80 \\
\hline & Capsule & Amperometry & GOx & Glucose & Diabetes & 25 \\
\hline
\end{tabular}

\section{lontophoresis-based sweat induction methods}

Noninvasive, continuous monitoring of alcohol levels in sweat is necessary for law enforcement personnel, the service/hospitality industry, or individuals to better manage alcohol consumption. To this end, many efforts have been dedicated to develop one-time use (i.e., disposable) temporary tattoo-based alcohol sensors via a cost-effective printing technique (e.g., screen printing) to print various functional inks (e.g., Prussian blue inks, conductive carbon inks) on a number of flexible substrates (e.g., papers, plastics ${ }^{42-47}$. Figure 2a (left panel) shows an example of a temporary tattoo-based alcohol sensor that enables the on-skin amperometric detection of ethanol in locally induced sweat through the transdermal delivery of a drug compound (e.g., pilocarpine) to help the sweat glands produce sweat ${ }^{42}$. As illustrated in Fig. 2a (middle panel), the sensor consists of a screen-printed iontophoretic component at the anode that can electrically induce the migration of pilocarpine across the skin at an applied current of $0.6 \mathrm{~mA}\left(0.2 \mathrm{~mA} \mathrm{~cm}^{-2}\right)$ to generate quantifiable localized sweat in a relatively short time of $5 \mathrm{~min}^{47}$. Subsequently, the amperometric detection of ethanol occurs at the cathode using a screen-printed Prussian blue (PB) transducer along with a chitosanimmobilized alcohol-oxidase ( $\mathrm{AOx}$ ) enzymatic electrode. The sensor exhibited a high sensitivity of $0.362 \pm$ $0.009 \mu \mathrm{A} \mathrm{mM}^{-1}$ with good linearity within an ethanol concentration range from 0 to $36 \mathrm{mM}$ (Fig. 2a, right panel). For on-body field testing in healthy participants, the sensor was wired with a custom-built flexible supporting electronic readout circuit for a wireless Bluetooth connection to a laptop or mobile device. This sensor was also able to conform to the skin and immediately detect iontophoretically extracted sweat with minimal risk of external contamination. The results also highlighted that the current response of this sensor was clearly distinct before and after the consumption of an alcoholic beverage. The approach that involves chemically inducing sweat followed by immediate electrochemical analysis may eliminate the need for conventional sweat induction approaches, such as exercise and sauna exposure, that are often inapplicable to people with mobility limitations or elderly people. Despite its promising application, there remains an opportunity to alleviate the side effects of the iontophoresis process, such as dryness, blistering, or irritation of the skin ${ }^{48}$.

\section{Microfluidic-based sweat collection methods}

A new class of thin and soft sweat sensors that include microfluidic channels has recently started to emerge, offering an advanced capability for the collection of sweat with minimal evaporation and contamination and it's subsequent in situ analysis. The microfluidic channels of these sensors capture sweat directly from the skin and then 


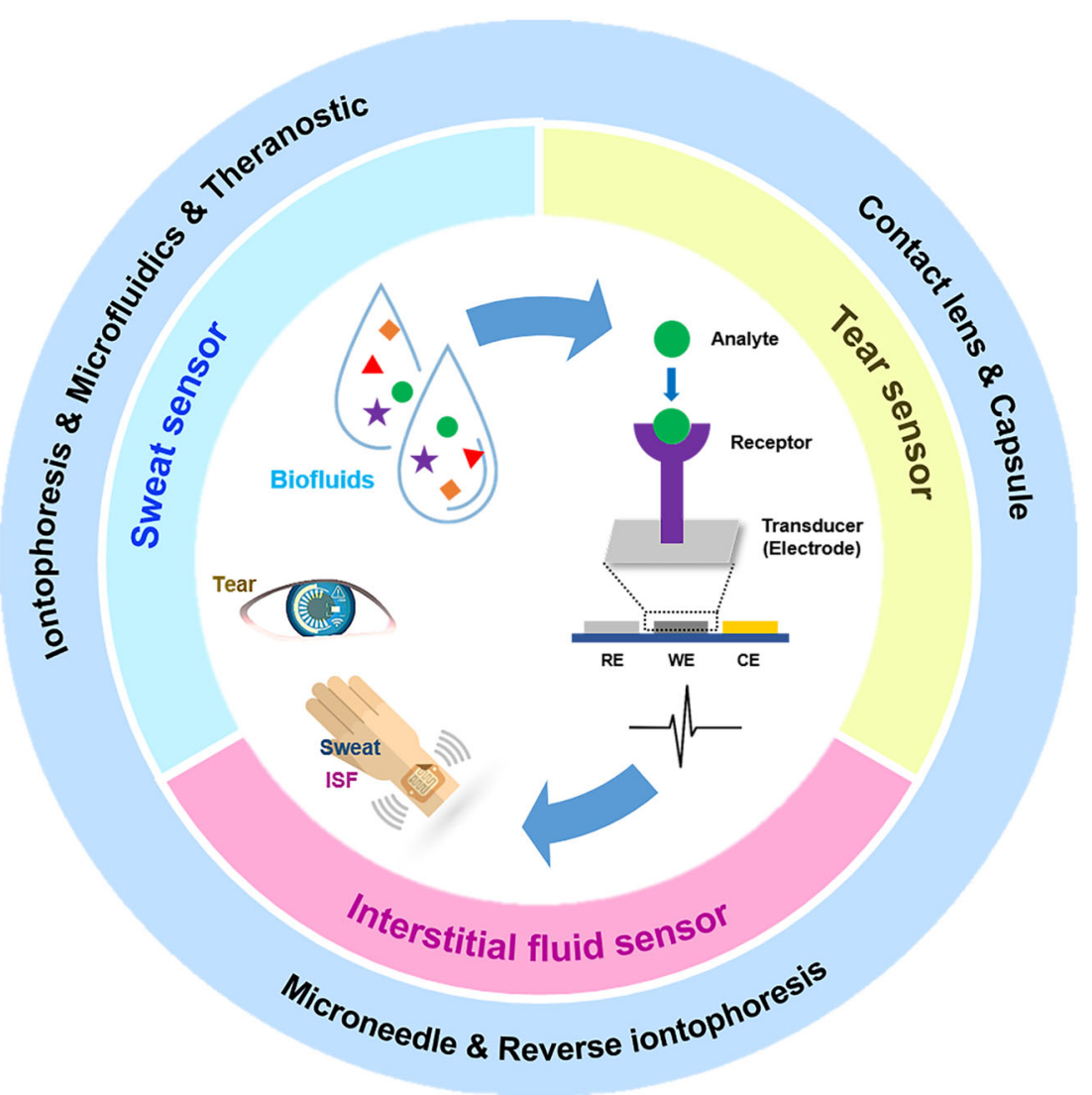

Fig. 1 Schematic diagram of wearable elecrochemical sensors. The sensors are classified into three groups according to their target body fluids such as sweat, interstitial fluid (ISF), and tears.

transport it through valves (i.e., a network of microchannels), separators, and reservoirs by exploiting the capillary force associated with perspiration ${ }^{49-51}$. To further facilitate capillary action, these microfluidic channels are often integrated with a sweat-absorbent layer (or sweat uptake layer) made from a porous hydrophilic structure such as a sponge and cellulose paper that is capable of effectively accumulating sweat ${ }^{52}$. The sophisticated design of these microfluidic channels can also prevent the rapid evaporation of sweat while increasing the temporal resolution of the sweating process. As a consequence, these microfluidic channels lead to a rich collection of sweat and thereby, in general, provide consistent measurement results at low sweat rates with minimal risk of dilution and external contamination of sweat. The microfluidic sweat sensors can be disposable by using cost-effective materials (e.g., silicone elastomers, plastics) and simple production methods (e.g., molding, $\mathrm{CO}_{2}$ laser-engraving techniques) without the need for personnel training and process optimization $^{50-55}$. Figure $2 \mathrm{~b}$ (left panel) shows an example of a microfluidic sweat sensor that enables the continuous monitoring of uric acid and tyrosine in sweat, which is particularly useful for patients suffering from gout ${ }^{56}$.
Gout is the most common inflammatory arthritis and affects more than 40 million people worldwide annually; it is typically characterized by elevated levels of uric acid and tyrosine that exceed physiological saturation thresholds. Thus, the noninvasive continuous monitoring of uric acid and tyrosine in sweat is highly desired and necessary for the personalized management of gout through uratelowering therapy or nutritional control. To this end, the microfluidic sweat sensor consists of (1) a multi-inlet microfluidic module for the dynamic sampling of sweat by minimizing its evaporation and contamination, (2) a chemical sensor that is capable of detecting low concentrations of uric acid and tyrosine using graphene due to its unique electrochemical properties arising from the fast electron mobility, high current density, and large surface area, and (3) additional physical sensors that are capable of simultaneously monitoring both the skin temperature and respiration rate of the wearer to further ensure appropriate and abundant analytical information. All of these components are fabricated on a polyimide sheet with medical tape using a $\mathrm{CO}_{2}$ laser-cutting machine. This sensor exhibited high sensitivities of $3.50 \mu \mathrm{A} \mu \mathrm{M}^{-1} \mathrm{~cm}^{-2}$ and $0.61 \mu \mathrm{A} \mu \mathrm{M}^{-1} \mathrm{~cm}^{-2}$ and low 


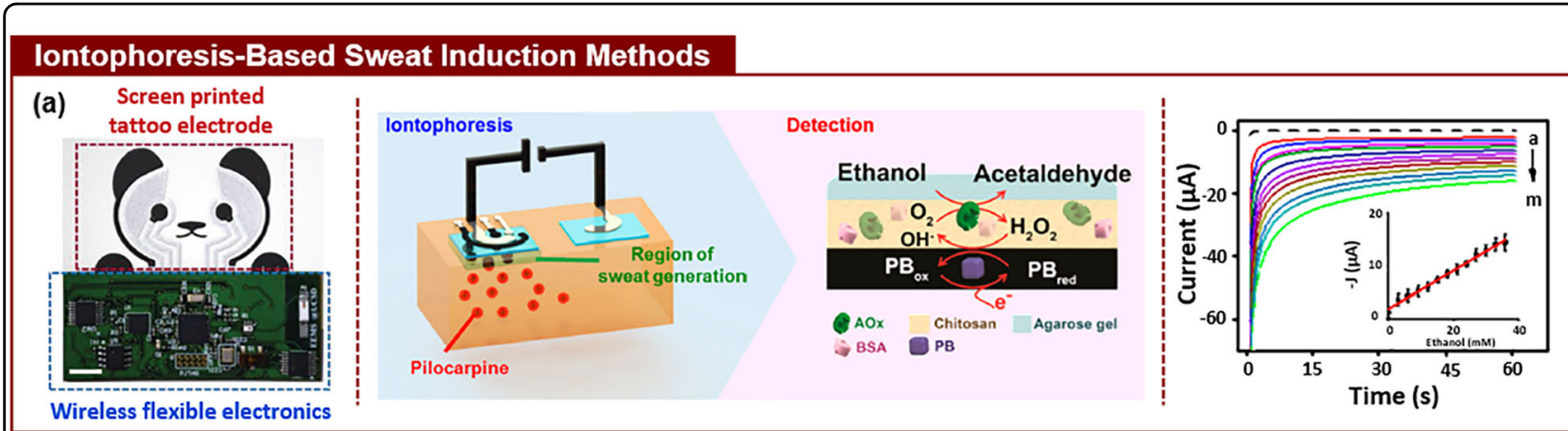

\section{Microfluidic Sweat Collection Methods}

(b)
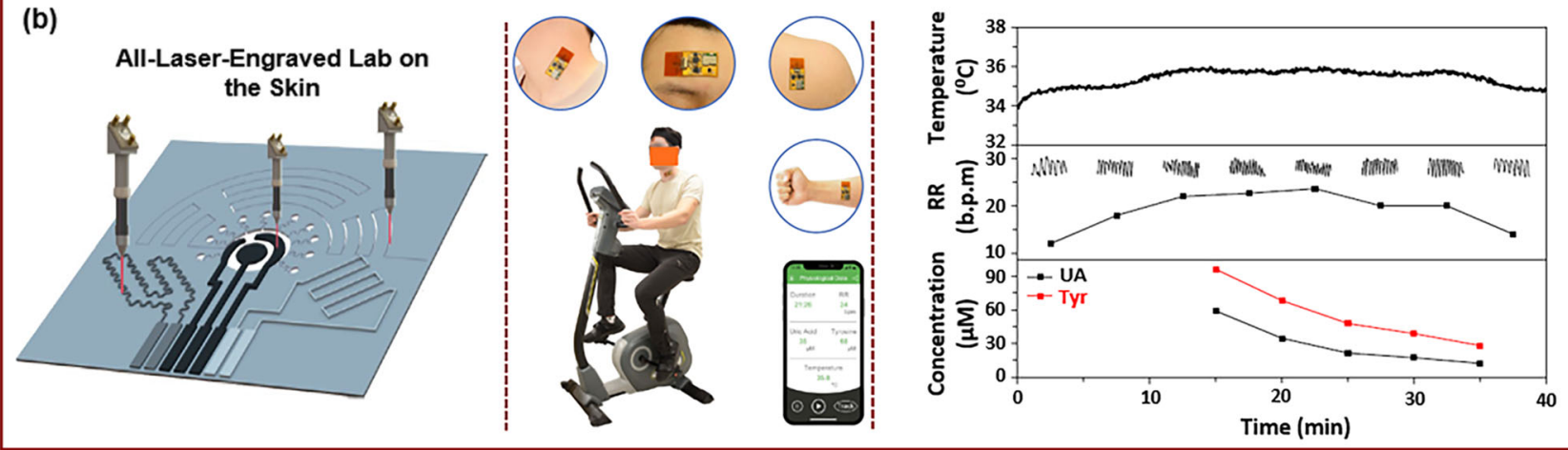

\section{Theranostic Sweat Sensors with Drug Delivery System}

(c)

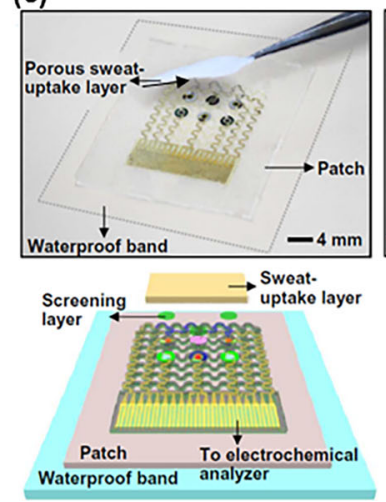

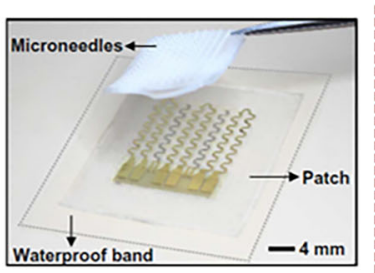
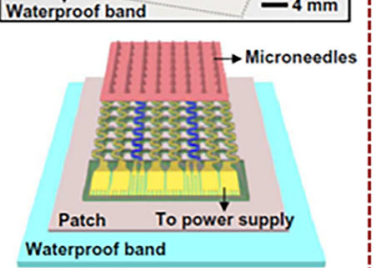
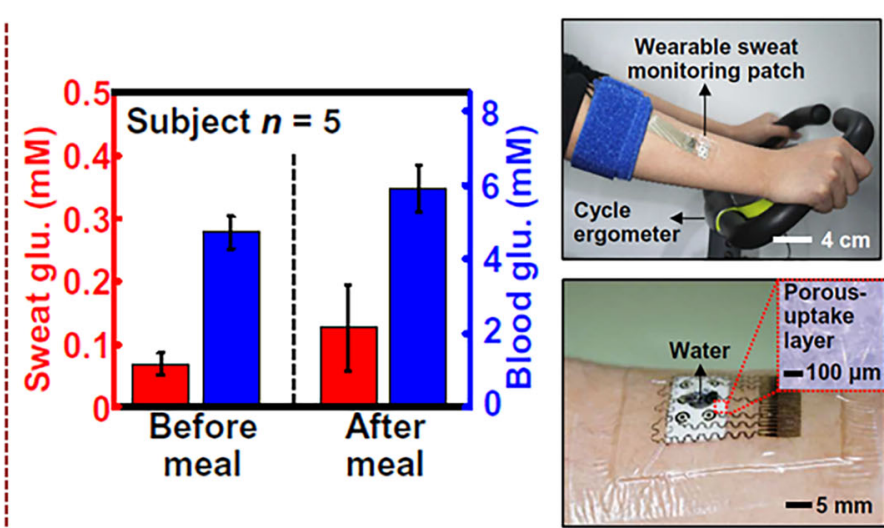

Fig. 2 Wearable sweat sensors. a, left: Image of a tattoo-like sensor integrated with a wireless unit. Middle: Schematic diagram of the constituents of an iontophoretic system and of the reagent layer and the process involving the amperometric sensing of ethanol on the working electrode. Right: Amperometric response of the tattoo-like sensor to increasing ethanol concentrations from $0 \mathrm{mM}$ to $36 \mathrm{mM}$. Reproduced with permission from ref. ${ }^{42}$. Copyright 2018, WILEY-VCH Verlag GmbH \& Co. KGaA, Weinheim. Reproduced with permission from ref. ${ }^{47}$. Copyright 2016, American Chemical Society. $\mathbf{b}$, left: The laser-engraved microfluidic sweat sensor. Middle: Photograph of a healthy subject wearing the sensor on different body parts. Right: Real-time, continuous, in situ measurement of the respiration rate (RR; measured in breaths per minute (b.p.m.)), temperature, and uric acid and tyrosine levels in sweat from the neck of a healthy subject during a constant-load stationary cycling exercise. Reproduced with permission from ref. ${ }^{56}$. Copyright 2019, Springer Nature. c, left: Photographs and schematic illustrations of a theranostic sweat sensor. Middle: Comparison of sweat and blood glucose concentrations before and after a meal. Right: Photographs of the subject using a cycle ergometer for sweat generation with the sensor placed on the subject's arm. Reproduced with permission from ref. ${ }^{59}$ under the terms of the Creative Commons Attribution-NonCommercial 4.0 International (CC BY-NC 4.0), Science Advances.

detection limits of $0.74 \mu \mathrm{M}$ and $3.6 \mu \mathrm{M}$ for the oxidation of uric acid and tyrosine at physiological concentrations, respectively. For pilot preclinical evaluations in patients with gout, the sensor was placed on a different part of the body (e.g., neck, forehead, forearm) and then connected to a custom-built wireless unit that is also capable of flexibly adhering to the skin (Fig. 2b, middle panel). The acquired data were transmitted via a Bluetooth connection to a 
mobile device for post analysis. The measurement results showed that the dynamic changes in uric acid in sweat before and after a purine-rich meal over a 7 -h period were well followed by the changes in serum uric acid with a high correlation coefficient of 0.864 (Fig. 2b, right panel). The results demonstrated the potential utility of this sensor for the precise adjustment of personalized doses in urate-lowering therapies for gout patients.

\section{Theranostic sweat sensors with a drug delivery system}

Diabetes is one of the most prevalent chronic diseases; it results in hyperglycemia and is caused by a deficiency in the production of insulin or resistance to insulin ${ }^{57}$. Failure to manage the blood glucose level of diabetes patients may lead to various severe diabetic complications, such as kidney disease, stroke, and even death. Thus, there is an urgent need to develop a method for the noninvasive, portable, and continuous monitoring of glucose levels throughout all instances of eating events to better control the dose of mealtime insulin. To maintain homeostasis in the body, another opportunity exists for the controlled feedback delivery of a precise dose of drugs (e.g., metformin) in response to the measured glucose level ${ }^{58,59}$. Figure $2 \mathrm{c}$ (left panel) shows an example of a theranostic sweat sensor that is integrated with a transdermal drug delivery system to improve the sweat-based management of diabetes patient $^{59}$. Specifically, this sensor is configured as a thin and stretchable (i.e., serpentine) layout to establish a conformal interface with the skin and thereby reliably maintain its performance during on-body movements (i.e., movements that result in skin deformations) ${ }^{60-62}$. A waterproof bandaid is used to prevent the delamination of the sensor from the skin. For sweat collection, a Prussian blue-deposited porous gold electrode is used along with multiple sweat uptake layers to obtain a quantifiable sweat sample $(\sim 1 \mu \mathrm{l})$ as well as a negatively charged porous Nafion layer to immobilize the enzymes (e.g., glucose oxidase (GOx)) and screen out negatively charged molecules (e.g., drug molecules that may be present in sweat and affect glucose monitoring). This sensor is also integrated with a series of other sensing elements that are capable of detecting the humidity, $\mathrm{pH}$, and temperature of sweat during the collection procedure via a poly(3,4-ethylenedioxyhiophene) (PEDOT) electrode, polyaniline (PANi)deposited working electrode, and a temperature-sensitive resistor, respectively. The humidity data are used to help the user estimate an appropriate starting point of sweat analysis, while the $\mathrm{pH}$ and temperature data are used to correct potential errors in the measurement of enzymebased glucose levels. For transdermal drug delivery, the sensor is also integrated with an array of thermoresponsive (hyaluronic acid) hydrogel microneedles coated with phase change materials containing testbed drugs (i.e., metformin; one of the widely used first-line drugs to treat type 2 diabetes). The melting temperature of the phase change materials remains above body temperature to hold the drugs without leakage prior to thermal activation using a heater (embedded in the sensor). The phase change of the materials occurs controllably at either $40^{\circ} \mathrm{C}$ or $45^{\circ} \mathrm{C}$, thereby enabling multistage drug delivery in response to the measured glucose level. For pilot preclinical evaluations in healthy human subjects, this sensor was connected to a custom-built portable electrochemical analyzer to power, control, and transmit wireless data via a Bluetooth connection. The measured sweat glucose levels showed a clear increase after a meal and showed a close correlation to the glucose levels obtained from simultaneous blood glucose measurements using a commercial glucose assay kit (Fig. 2c, right panel). In vivo evaluations in a diabetic mouse model were also conducted to show the feasibility of possible closed-loop therapies to controllably suppress the blood glucose level through the feedback delivery of metformin. Further technological developments are still required for the extended application of these closed-loop therapies beyond glucose ${ }^{63}$.

\section{Wearable ISF sensors}

ISF is a thin layer of fluid surrounding blood vessels and cells throughout the body and is formed by blood transcapillary filtration and cleared by lymphatic vessels ${ }^{16}$. ISF is a rich source of biomarkers due to its similarity to blood in terms of protein composition, small-molecule metabolite composition, and RNA profile. In addition, ISF may provide a better indicator of local tissue events than blood due to its coagulation-free characteristics and high sensitivity to changes in local tissues. Many minimally invasive sampling methods of ISF have been developed through the innovation of various types of microneedles typically made from polymers that can penetrate into the skin and then induce a suitable driving force to transport ISF out of the body ${ }^{64-66}$. In parallel, needleless (or noninvasive) sampling methods of ISF have also been developed by exploiting ultrasound, lasers, or reverse iontophoresis ${ }^{46}$. Of these methods, reverse iontophoresis is the most prevalent due to its simplicity, which involves applying a low electric current across the skin to drive biomarkers out of the blood vessels and transport them to the skin surface. For the in situ analysis of the extracted ISF on the skin (which is highly desired because the metabolites in ISF vary considerably over time), several attempts have been dedicated to integrating microneedles with a wearable (i.e., on-skin) electrochemical sensor for the simultaneous collection and analysis of ISF, followed by wireless transmission of the measured data for post analysis.

\section{Microneedle-based ISF sensors}

The use of a vertically aligned array of microneedles has emerged as a promising method that allows access to 
intratissue space in a minimally invasive manner and has enabled many effective strategies for the sampling of ISF. Microneedles with a depth of $<200 \mu \mathrm{m}$ are capable of piercing through the epidermis or the upper dermis layer with minimal pain, allowing for either the collection of a quantifiable amount of ISF by capillary action or the direct measurement of ISF by utilizing the microneedles as recording electrodes ${ }^{66,67}$. These microneedles can be monolithically integrated with a wearable electrochemical sensor for continuous in situ analysis of the metabolites in ISF with a short sampling time to minimize the risk of external contamination. Figure 3a (top panel) shows an example of a microneedle-based ISF sensor that is particularly tailored for the continuous direct measurement of the levodopa concentration in ISF, which is useful for patients with Parkinson's disease ${ }^{67}$. Levodopa has served as the benchmark antiparkinsonian drug for many decades. For effective management of Parkinson's disease, the dose of levodopa must be controlled to avoid any negative consequences in which low doses lead to parkinsonism, anxiety, and depression. Thus, there is a critical opportunity to develop a wearable sensor to provide individualized information associated with the concentration of levodopa in a timely manner. To this end, this sensor consists of three microneedles filled with different carbon pastes to form (1) an enzyme-modified working electrode, (2) an unmodified working electrode transducer, and (3) an $\mathrm{Ag} / \mathrm{AgCl}$ reference electrode. Each microneedle has a hole through which ISF is transported. This sensor provides a dual-mode sensing capability in which independent electrochemical measurements (involving redox and biocatalytic processes) occur at the two working electrodes. Specifically, direct (nonenzymatic) anodic detection of levodopa occurs at the unmodified working electrode using square-wave voltammetry (SWV), while tyrosinase (TYR)-based biocatalytic detection occurs at the neighboring enzyme-modified working electrode via chronoamperometric measurement of the corresponding dopaquinone product. For pilot field testing in a mouse model, this sensor was connected to a portable electroanalyzer for wireless data transmission (Fig. 3a, bottom panel), showing favorable analytical performance in the multimodal sensing of levodopa upon penetration of the microneedles through mouse skin, with high sensitivity $\left(0.048 \mathrm{nA} \mu \mathrm{M}^{-1}, \quad R^{2}=0.999\right)$, selectivity, and stability (standard deviation of $2 \%$ ) along with a wide linear dynamic range (between 20 and $300 \mu \mathrm{M}$ ).

Figure $3 \mathrm{~b}$ (top panel) shows another example of a microneedle-based wearable ISF sensor that enables the continuous assessment of the subcutaneous alcohol concentration in $\mathrm{ISF}^{68}$. This sensor aims to provide an easierto-use and more reliable means to evaluate alcohol consumption compared to conventional methods for blood alcohol sampling. This sensor consists of three pyramidal microneedles with apertures through which a Pt or Ag wire is inserted to form working, counter, and reference electrodes. Each microneedle was used as an electrochemical electrode to directly measure the ISF. The Ptwire-integrated microneedle (working) electrode is functionalized with an electropolymerized poly(o-phenylenediamine) (PPD) film along with an AOx enzyme immobilized with a chitosan matrix and an additional coating of a Nafion film. The multilayer PPD/Nafion coating serves as a permselective/protective barrier to prevent potential interferents from coming into contact with the surface of the microneedles. The Pt electrode transducer is capable of detecting the hydrogen peroxide $\left(\mathrm{H}_{2} \mathrm{O}_{2}\right)$ generated by the AOx-catalyzed oxidation of ethanol, producing an oxidation current correlated to the target ethanol concentration. This sensor showed a high sensitivity of $0.062 \mathrm{nA} \mathrm{mM}^{-1}$ in chronoamperometric measurements using $0.1 \mathrm{M}$ phosphate buffer solution $(\mathrm{pH}=7.0)$ with ethanol up to $80 \mathrm{mM}$ at a sampling rate and applied working potential of $35 \mathrm{~s}$ and $0.6 \mathrm{~V}$ (versus $\mathrm{Ag} / \mathrm{AgCl}$ ), respectively. As shown in Fig. 3b (bottom panel), the chronoamperometric response of the microneedles was measured ex vivo upon their penetration through mouse skin at $0.6 \mathrm{~V}$ (versus $\mathrm{Ag} / \mathrm{AgCl}$ ) after $100 \mathrm{~s}$ of incubation in an artificial ISF solution. The results clearly showed that the concentration of ethanol in the ISF solution increased linearly in $25 \mu \mathrm{M}$ steps, demonstrating the feasibility of continuously monitoring the subcutaneous alcohol level in ISF.

\section{Reverse-iontophoresis-based ISF sensors}

Reverse iontophoresis is a technique that involves applying a potential difference across the skin, leading to an electro-osmotic flow of ions (e.g., $\mathrm{Na}^{+}$) as charge carriers due to the nature of human skin, which has a net negative charge at physiological $\mathrm{pH}$. As a consequence, neutral molecules in ISF, including glucose, are extracted to the outermost surface of the skin. Subsequently, in situ enzymatic electrochemical detection of the as-extracted biomarkers can be performed on the skin using a wearable ISF sensor. Figure 3c shows an example of a reverseiontophoresis-based ISF sensor that incorporates (1) Ag/ $\mathrm{AgCl}$ reverse-iontophoresis electrodes (along with an agarose hydrogel coating) to extract glucose from ISF and then deliver glucose close to the working and counter/ reference electrodes and (2) a GOx-modified Prussian blue transducer for subsequent amperometric sensing at a low potential ${ }^{46}$. In pilot in vitro testing, the chronoamperometric response of the sensor showed good linearity over a glucose concentration range of 0 to $100 \mu \mathrm{M}$. The sensor exhibited a high sensitivity of $23 \mathrm{nA} \mu \mathrm{M}^{-1}$ and a sufficient level of specificity for detecting a micromolar concentration of glucose in the presence of common interfering electroactive species, such as ascorbic acid, 


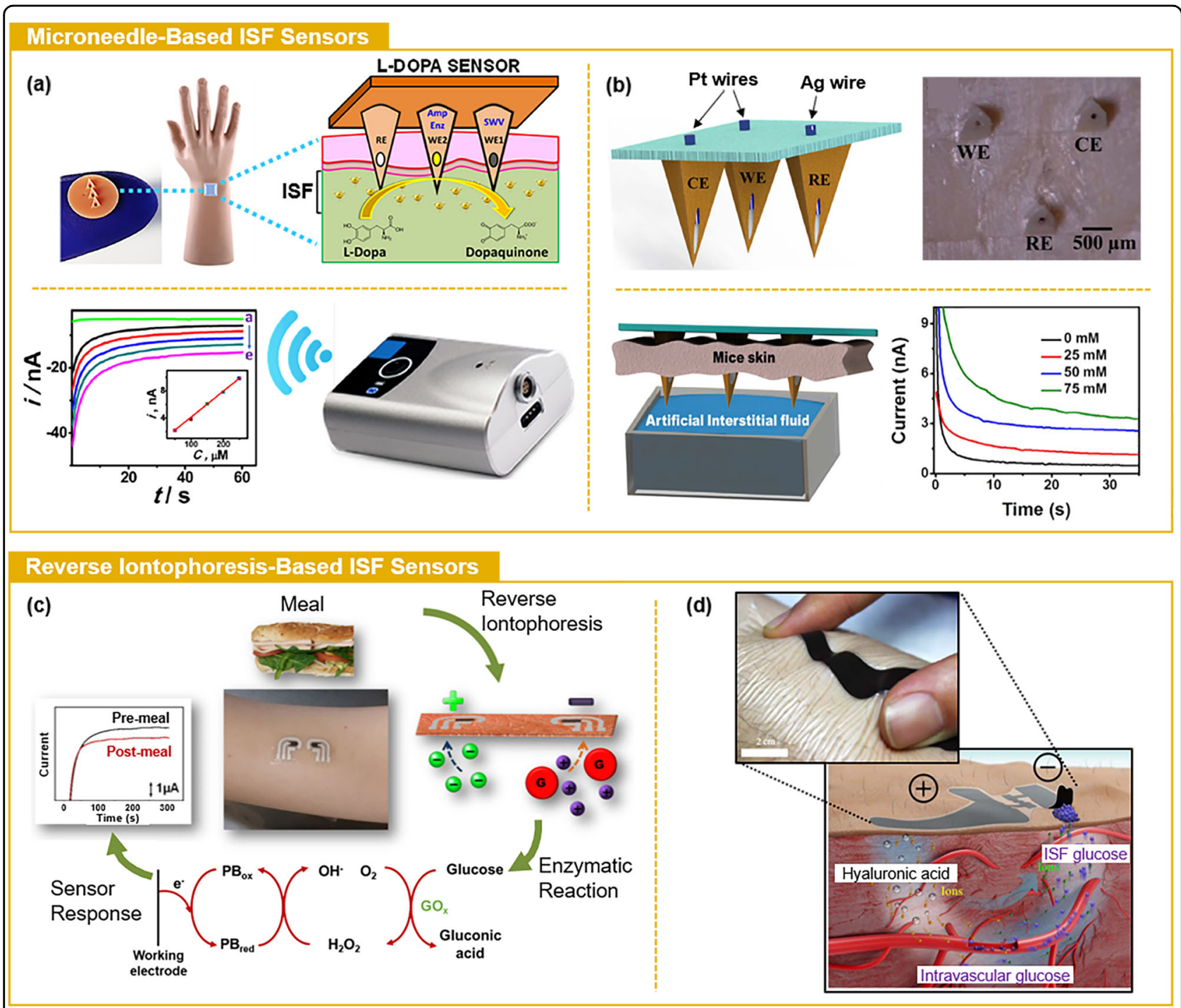

Fig. 3 Wearable interstitial fluid (ISF) sensors. a Schematic illustration of a mannequin hand wearing a microneedle sensor and the microneedle sensor for the monitoring of ISF levodopa. Chronoamperometry responses of ISF levodopa from 50 to $250 \mu \mathrm{M}$. A portable wireless electroanalyzer enabled wireless data transmission to a mobile device. Reproduced with permission from ref. ${ }^{67}$. Copyright 2019, American Chemical Society. b Schematic illustration and photograph of the microneedles penetrated through mouse skin for the real-time detection of alcohol in artificial ISF. Reproduced with permission from ref. ${ }^{68}$. Copyright 2017, Elsevier. c Illustration of the reverse-iontophoresis-based sensing process for glucose in ISF. Reproduced with permission from ref. ${ }^{46}$. Copyright 2014, American Chemical Society. d Schematic illustration of electrochemical twin channels (ETCs) for the improved transport of intravascular glucose into ISF. The inset photograph highlights the ultrathin sensor platform. Reproduced with permission from ref. ${ }^{69}$ under the terms of the Creative Commons Attribution-NonCommercial 4.0 International (CC BY-NC 4.0), Science Advances.

uric acid, and acetaminophen. Subsequently, in vivo onbody testing was performed for healthy participants in a fasting state. A constant current of $0.2 \mathrm{~mA} \mathrm{~cm}^{-2}$ was applied between the electrodes for 10 min to extract the ISF to the skin, followed by the amperometric recording of the glucose response at an applied potential of $-0.1 \mathrm{~V}$ (vs $\mathrm{Ag} / \mathrm{AgCl}$ ) for $5 \mathrm{~min}$. The extraction and detection processes of ISF glucose were repeated before and after the consumption of a carbohydrate-rich meal, while the participants were asked to wait for $5 \mathrm{~min}$ prior to postmeal measurements to compensate for the intrinsic density time lag of 15-20 min between the glucose in the blood and that in ISF. The measurement results clearly displayed a distinct increase in the postmeal glucose level compared to the fasting state, along with a close correlation to the levels obtained from simultaneous fingerstick blood glucose measurements using a commercial glucose strip (Accu-Chek Aviva Plus).

Figure 3d shows another example of a reverseiontophoresis-based ISF sensor using electrochemical 
twin channels (ETCs) that are designed to efficiently drive intravascular blood glucose out of the subcutaneous vessel and transport it to the skin surface ${ }^{69}$. This sensor is made by stacking multiple thin films from the bottom, including polymethylmethacrylate (PMMA) (80 nm thick), polyimide ( $1.6 \mu \mathrm{m}$ thick), an Au thin film ( $100 \mathrm{~nm}$ thick), an electrochemically deposited transducer ( $51.8 \mathrm{~nm}$ thick), and a GOx immobilization layer ( $1 \mu \mathrm{m}$ thick), which results in a total thickness of $\sim 3 \mu \mathrm{m}$. The ultrathin nature of this sensor enables it to make intimate contact with the skin to further facilitate the extraction and detection processes of intravascular blood glucose with improved sensitivity (130.4 $\mathrm{mA} \mathrm{mM}^{-1}$ ) and an improved signal-tonoise ratio. For the extraction of intravascular blood glucose, a flexible biocompatible paper battery was first placed on the skin for $20 \mathrm{~min}$ to generate the subcutaneous ETCs that lead to (1) the penetration of hyaluronic acid into ISF at the anode, (2) the refiltration of intravascular blood glucose from the vessel, and then (3) the reverse iontophoresis of glucose to the skin at the cathode. Under the paper battery anode, extra hyaluronic acid was added not only to increase the osmotic pressure of ISF but also to promote intravascular blood glucose refiltration at the arterial ends. As a consequence, the intravascular blood glucose was efficiently extracted at a high rate with an improved correlation to the real blood glucose level compared with other methods because the extracted intravascular blood glucose also contains the glucose level that previously existed in blood. For the subsequent detection process, the wearable ISF sensor was then firmly attached to the cathode-contacting area of the skin. The measurement results obtained from pilot on-body field testing with healthy participants were well matched with those obtained using a commercial fingerprick glucometer. There was a 1-h time lag in these measurements, which was caused by the intrinsic density time lag of 15-20 min between the glucose in the blood and that in ISF as well as the measurement operation time of $\sim 25 \mathrm{~min}$.

\section{Wearable tear sensors}

The human eye is much softer and smaller and much more sensitive to pain than the skin; therefore, there are greater challenges in the continuous monitoring of tears at the corneal surface ${ }^{41,70}$. Over many decades, the biomedical science and engineering community has expressed a great desire to use contact lenses as eye-wearable sensors owing to their noninvasive accessibility to the corneal surface and tears at the same time $\mathrm{e}^{71,72}$. However, current contact lenses serve to either correct vision problems or deliver ocular drugs passively over a given time. Recent technological developments have enabled the emergence of smart contact lenses, including Google's smart contact lenses, for providing the capability to continuously monitor glucose levels in the wearer's tears $^{41,72}$. Although these contributions are intriguing to those interested in rapidly introducing science-fiction-like ideas into the market, several key challenges remain ${ }^{73}$. Specifically, most existing smart contact lenses are fabricated on custom-made contact lenses comprised of materials that can tolerate the conditions used in the fabrication of microsensors and electronics ${ }^{38}$. Their fabrication processes are typically carried out using transparent plastics (e.g., polyethylene terephthalate (PET) $)^{74,75}$ or a combination of polymers (e.g., PMMA, SU8 resin) ${ }^{32}$. Consequently, these devices suffer from limited oxygen transmissibility (leading to short-term wearability), wettability (leading to eye dryness and irritation), and softness and ergonomic curvature (leading to wearer discomfort), thereby impeding their practical implementation. More recently, many ongoing research efforts have enabled the phenomenal success of fabricating a range of flexible sensors on custom-built contact lenses made of silicone elastomers or Parylene- $\mathrm{C}^{76}$. These sensors offer enhanced on-eye transparency, oxygen transmissibility, and flexibility via the use of functional nanomaterials (e.g., graphene and nanowires) ${ }^{77-80}$. Their implementation in clinical practice is in progress to further improve the mechanical reliability (for lens handling, fitting, cleaning, and inadvertent eye rubbing) and chemical stability (for long-term lens storage and multiple disinfection cycles). In this section, we introduce recent progress in eyewearable sensors tailored for the continuous monitoring of tear biomarkers.

\section{Contact lens-based tear sensors}

Contact lenses are very popular and the most common eye-wearable platform, with more than 70 million users worldwide $^{72}$. When inserted into the eye, contact lenses naturally touch the cornea in a noninvasive manner, holding great potential to serve as an ideal sensor platform for the continuous monitoring of tear biomarkers ${ }^{41,73}$. Figure $4 \mathrm{a}$ shows an example of a contact lens-based tear sensor that is wirelessly controlled to detect both tear glucose levels and intraocular pressure $(\mathrm{IOP})^{78}$. This lens incorporates a number of nanomaterials, including graphene and $\mathrm{Ag}$ nanowires, to provide excellent transparency $(>91 \%)$ and stretchability $(\sim 25 \%)$, therefore promoting on-eye safety and vision. The sophisticated combination of these nanomaterials leads to the construction of several electronic components, including (1) an array of field-effect transistors (FETs) for glucose sensors, in which the graphene-based channels are immobilized with GOx using a pyrene linker by $\Pi-\Pi$ stacking and in which Ag nanowire-based source/ drain electrodes are passivated with SU-8; (2) an inductance-capacitance (LC) resonance circuit for an IOP sensor in which a soft elastomer is sandwiched 


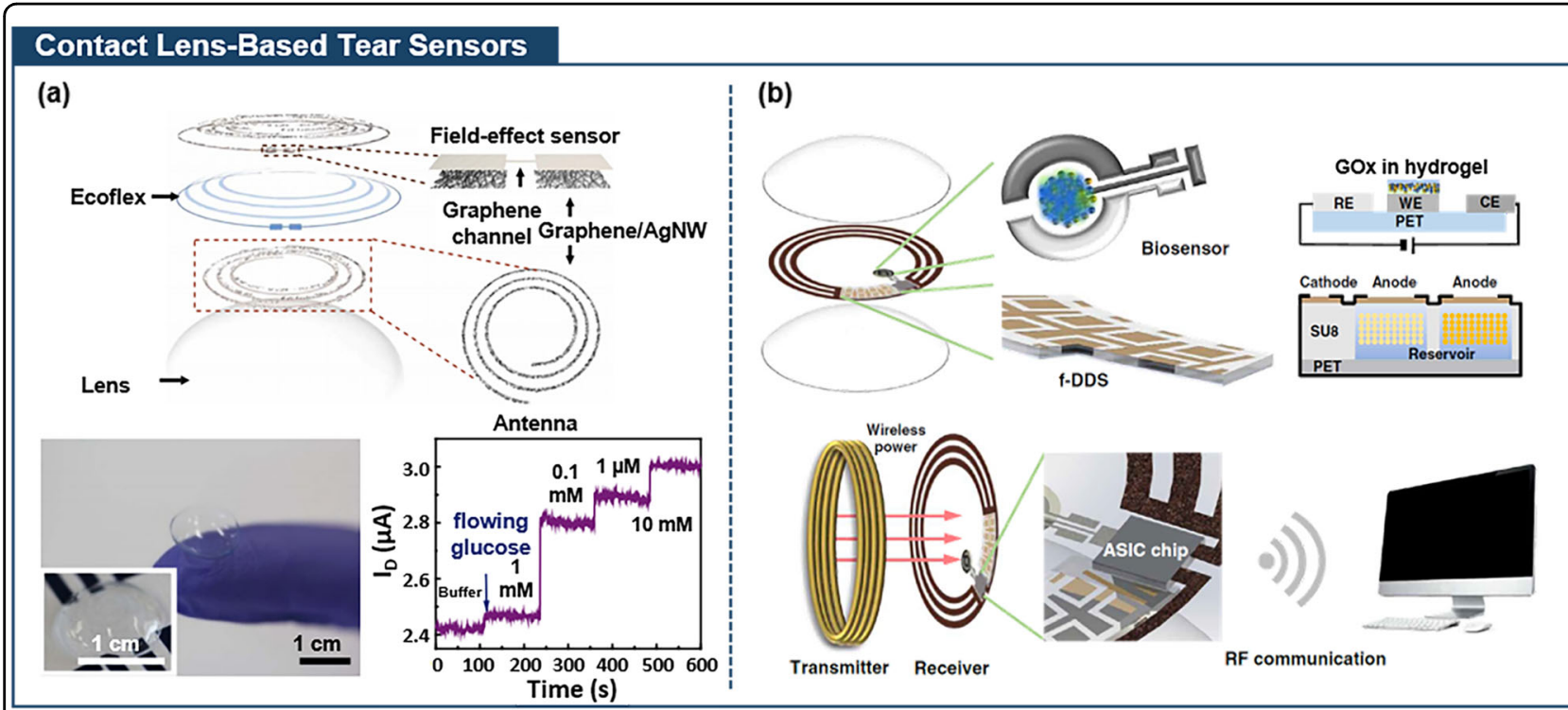

\section{Capsule-Based Tear Sensors}

(c)

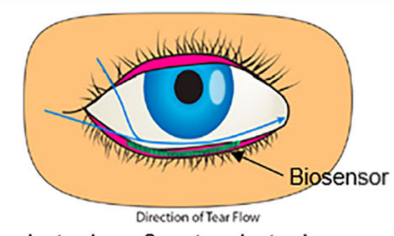

Working electrode Counter electrode
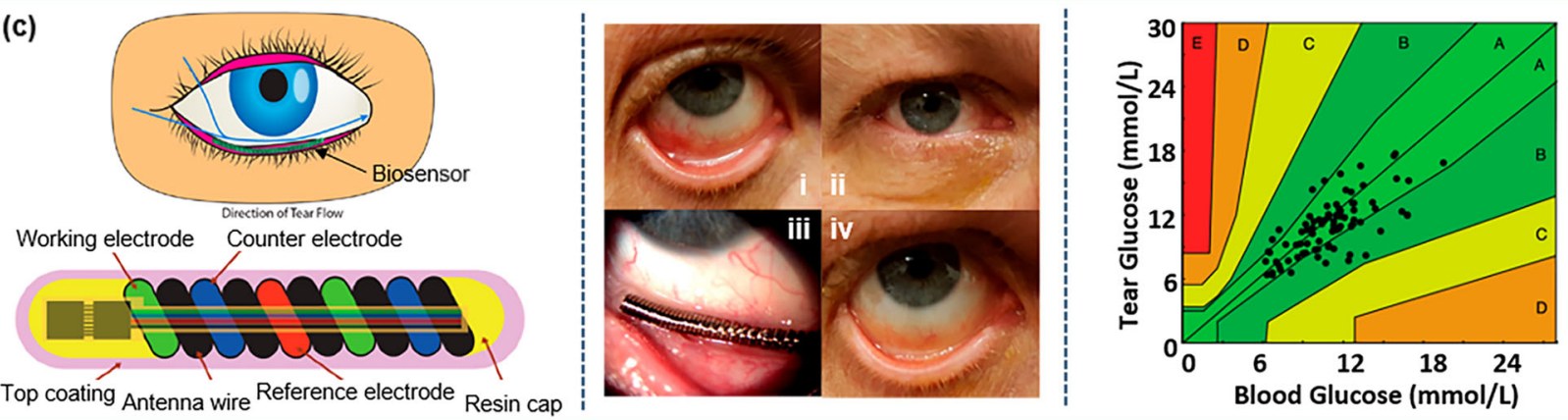

Fig. 4 Wearable tear sensors. a Schematic illustration and photograph of a contact lens-type sensor for the continuous monitoring of tear glucose levels. Reproduced with permission from ref. ${ }^{78}$ under the terms of the Creative Commons Attribution-NonCommercial 4.0 International (CC BY-NC 4.0), Springer Nature. b Schematic illustration of the contact lens-type multimodal sensor that is integrated with an on-board integrated circuit chip for wireless connection. Reproduced with permission from ref. ${ }^{76}$ under the terms of the Creative Commons Attribution-NonCommercial 4.0 International (CC BY-NC 4.0), Science Advances. c, left: Schematic illustration of the capsule-based tear sensor for the continuous monitoring of tear glucose levels. Middle: Photographs of human eyes in phase II clinical trial for (i) a baseline evaluation, (ii) middle of the trial, (iii) location of the sensor in the inferior conjunctival fornix, (iv) after the trial. Right: Consensus grid acquired from six clinical subjects wearing the sensor for $4.5 \mathrm{~h}$. Reproduced with permission from ref. ${ }^{25}$. Copyright 2018, American Chemical Society.

between the Ag nanowire-based electrodes; and (3) a resistance-inductance-capacitance (RLC) resonance circuit in which the impedance of the radio frequency circuitry is matched for efficient wireless powering. The sensing mechanism of tear glucose is that GOx promotes the oxidation of glucose in tears to gluconic acid as well as the reduction of water to $\mathrm{H}_{2} \mathrm{O}_{2}$. The reducing agent (i.e., $\mathrm{H}_{2} \mathrm{O}_{2}$ ) is subsequently oxidized to produce oxygen, protons, and electrons, leading to an increase in the drain current of the FETs with respect to the increased glucose level in tears. The sensing mechanism is that an increase in IOP gives rise to an increase in the capacitance and inductance of the sensor by thinning the soft dielectric layer (e.g., Ecoflex) and laterally stretching the induction coil. These changes result in a detectable shift in the resonance frequency. Initial benchtop tests revealed that the glucose sensor detected concentrations of artificial tear fluids as low as $1 \mu \mathrm{M}$ with a signal-to-noise ratio of 7.34 at $1 \mu \mathrm{M}$, which is nearly tenfold higher than that of the previously reported counterpart devices made of metallic thin film electrodes. The IOP sensor also showed a high sensitivity of $2.64 \mathrm{MHz} \mathrm{mm} \mathrm{Hg}{ }^{-1}$ in a physiologically relevant range from 5 to $50 \mathrm{~mm} \mathrm{Hg}$. Pilot in vivo tests in rabbit eyes showed that this lens detected a change in tear glucose levels after $2 \mathrm{~h}$ of feeding the rabbits without any sign of irritation under blinking or eyeball movements.

Figure $4 \mathrm{~b}$ shows an example of a theranostic contact lens device that allows both the continuous monitoring of tear glucose levels and the controlled triggering of 
therapeutic ocular drugs to treat diabetic retinopathy ${ }^{76}$. Specifically, this lens consists of (1) a real-time amperometric sensor, (2) a self-regulated pulsatile drug delivery system, (3) a resonant inductive wireless energy transfer unit, (4) a complementary integrated circuit (IC)-based microcontroller chip with a power management unit (PMU), and (5) a remote radio frequency (RF) communication system. All of these electronic components are embedded inside a custom-built silicone hydrogel contact lens with a diameter of $14 \mathrm{~mm}$, a thickness of $200 \mu \mathrm{m}$, and a radius curvature of $8.0 \mathrm{~mm}$, along with an equilibrium water content of $33.6 \%$. The amperometric sensor is configured into working (i.e., Pt), counter (i.e., Pt), and reference (i.e., $\mathrm{Ag} / \mathrm{AgCl}$ ) electrodes; the working electrode is coated with a mixed solution of bovine serum albumin (BSA), poly(vinyl alcohol) (PVA), chitosan, and immobilized GOx with glutaraldehyde. The drug delivery system consists of multiple SU-8 reservoirs with a single dimension of $1.5 \mathrm{~mm} \times 3 \mathrm{~mm} \times 130 \mu \mathrm{m}$, each of which is covered with a defect-free $\mathrm{Au}$ anode membrane. The $\mathrm{Au}$ membranes can be selectively dissolved in the form of $\mathrm{AuCl}_{4}{ }^{-}$within $40 \mathrm{~s}$ in the presence of tears via the use of remote control to apply an electrical potential of $1.8 \mathrm{~V}$. The in vivo assessment of this lens in the eye of a diabetic rabbit demonstrated the capability of continuously detecting tear glucose levels and controllably triggering ocular drugs, all in a wirelessly controlled manner. The wireless monitoring of the current changes occurred within a physiologically meaningful range from 0 to $50 \mathrm{mg} \mathrm{dl}^{-1}$ before and after the injection of insulin. The on-demand delivery of a testbed therapeutic drug (e.g., antiangiogenic genistein) was also achieved through the cornea to the retina with a comparable therapeutic effect to conventional treatments (e.g., intravitreal injection of Avastin) on diabetic retinopathy. Importantly, no significant amount of heat was generated during the entire period of the wireless monitoring and drug delivery processes to ensure thermal safety.

\section{Capsule-based tear sensors}

Despite their promising applications, none of the existing contact lens-based sensors have been successfully reported in long-term clinical trials in the human eye. In addition, the remaining difficulty is an achievement of painlessly accessing the corneal surface and operating without side effects ${ }^{81}$. These circumstances have led to the development of capsule-based tear sensors as an alternative to contact lens-based devices, which can be unobtrusively worn under the lower eyelid for the continuous monitoring of tear glucose levels. Figure 4c (left panel) shows a commercial-grade capsule sensor (e.g., the NovioSense glucose sensor) that is configured into a flexible spring-shaped coil ${ }^{25}$. It fits well in the pocket of the inferior conjunctival fornix (near the meibomian glands) where a high volume of tears is collected. Upon insertion, the capsule sensor is unnoticeable due to its anatomical position (Fig. 4c, middle panel). The capsule contains (1) a spring-shaped coil made of Pt/Ir working electrodes and a polyesterimide/stainless steel insulator (15 $\mathrm{mm}$ long and $1.3 \mathrm{~mm}$ in diameter) to serve as an antenna for wireless power transfer, (2) a pair of microchips for wireless data transfer, and (3) an encapsulation package made of a hydrophilic polysaccharide-based hydrogel for comfort while wearing. The encapsulation package also includes immobilized GOx to serve as an enzymatic sensing element that can convert glucose and oxygen into gluconolactone and $\mathrm{H}_{2} \mathrm{O}_{2}$. The reducing agent (i.e., $\mathrm{H}_{2} \mathrm{O}_{2}$ ) is subsequently oxidized and detected on the working electrode via chronoamperometric measurements. Comprehensive preclinical and clinical studies were conducted in sheep and in patients with type 1 diabetes mellitus (phase II clinical trials), respectively. As shown in Fig. 4c (right panel), the results of the clinical trials highlighted a close correlation of the measured tear glucose levels with respect to the dynamic change in blood glucose levels (i.e., $>92 \%$ of the data fell into the A and $\mathrm{B}$ regions of the Clarke error grid) that were obtained using a commercial continuous glucose monitoring (CGM) product (e.g., Dexcom G4 and Abbott FreeStyleLibre). In addition, the capsule sensor had superior performance to a currently FDA-approved, skinmountable ISF sensor (e.g., Abbott FreeStyleLibre). This capsule sensor showed no acute response throughout the clinical trial timeframe, and additional large-cohort clinical trials are needed to confirm its utility.

\section{Conclusions and future prospects}

Recent attempts for the integration of electrochemically active materials with stretchable sensor platforms have substantially contributed to the emerging field of wearable health monitoring devices, particularly by enabling the continuous measurement of biomarkers in body fluids such as sweat, ISF, and tears. These stretchable and wearable sensors can analytically capture electrochemical changes in response to biological events. The thin, soft, and deformable nature of these sensors helps to establish a highly intimate and unobtrusive interface with a specific body part, such as the skin and eye, therefore enabling the long-term reliable monitoring of patients even during onbody movements in ambulatory care settings. Demonstrations of these sensors in the minimally invasive collection and in situ analysis of body fluids show clinical promise in the management of many metabolic diseases, such as diabetes, gout, and Parkinson's disease.

These wearable electrochemical sensors require viable power sources that also demand mechanical flexibility and excellent conformability to the skin ${ }^{82}$. Attempts have been made to use lithium-ion polymer batteries, triboelectric 
nanogenerators, and supercapacitors to demonstrate the practical applications of wearable electrochemical sensors ${ }^{4,83,84}$. Recent attempts to use biofuel cells that are capable of converting energy via redox reactions from biofluids to electrical energy open up a new opportunity for the biofuel cells to act as self-powered electrochemical sensors ${ }^{85,86}$. Biofuel cells provide unique advantages compared to other energy harvesting devices in terms of biocompatibility and auto-power features from biofluids ${ }^{87}$.

Some of these sensors have nearly commercial-grade performance in terms of sensitivity, selectivity, and response time, while their clinical correlations have been proven through comprehensive preclinical and clinical studies in both animals and humans. These sensors are also remote controlled via portable units for wireless data transfer, which facilitates further exploration of the possible home-based telemonitoring of patients from a distance. Telemonitoring intervention reduces the burden on the patients for whom frequent clinical visits are a challenge, especially for those who have limited mobility or live in rural areas. This aspect has become increasingly important due to the ongoing coronavirus (COVID-19) pandemic, which has also fueled rapid growth in this field.

The remarkable innovation of these wearable electrochemical sensors provides important insights into future extensive clinical trials to validate the effectiveness and efficacy of these sensors in the management of patients across a wide range of age groups and diagnoses. Additional cross-disciplinary research and development of these sensors will help to further tailor their mechanical, electrochemical, and biological properties for specific clinical needs with a clear path toward their immediate implementation in clinical practice. These efforts also need to focus on further improving the sensitivity and selectivity of these sensors in terms of the detection of low concentrations of target analytes that can vary according to the population, time, and body part under different ambient conditions. In turn, the results will better correlate with those under actual physiological conditions. Other future opportunities exist in the integration of multimodal sensors (i.e., simultaneous monitoring of multiple biomarkers) with artificial intelligence and/or machine learning tools to learn from the massive amounts of data collected for advanced postanalysis capability.

\section{Acknowledgements}

The authors acknowledge the funding support from the National Science Foundation (NSF) (CMMI-1928784, Program Manager: Dr. Khershed P. Cooper) and Eli Lilly and Company (20122871).

\section{Author details}

'Weldon School of Biomedical Engineering, Purdue University, West Lafayette, IN 47907, USA. ${ }^{2}$ School of Mechanical Engineering, Purdue University, West Lafayette, IN 47907, USA. ${ }^{3}$ School of Materials Engineering, Purdue University, West Lafayette, IN 47907, USA
Conflict of interest

The authors declare that they have no conflict of interest.

\section{Publisher's note}

Springer Nature remains neutral with regard to jurisdictional claims in published maps and institutional affiliations.

Received: 27 September 2020 Revised: 18 November 2020 Accepted: 3 December 2020.

Published online: 8 March 2021

\section{References}

1. Chu, B., Burnett, W., Chung, J. W. \& Bao, Z. Bring on the bodyNET. Nature 549, 328-330 (2017).

2. Ray, T. R. et al. Bio-integrated wearable systems: a comprehensive review. Chem. Rev. 119, 5461-5533 (2019)

3. Someya, T., Bao, Z. \& Malliaras, G. G. The rise of plastic bioelectronics. Nature 540, 379-385 (2016).

4. Gao, W. et al. Fully integrated wearable sensor arrays for multiplexed in situ perspiration analysis. Nature 529, 509-514 (2016).

5. Yang, Y. \& Gao, W. Wearable and flexible electronics for continuous molecular monitoring. Chem. Soc. Rev. 48, 1465-1491 (2019).

6. Yu, Y., Nyein, H. Y. Y., Gao, W. \& Javey, A. Flexible electrochemical bioelectronics: the rise of in situ bioanalysis. Adv. Mater. 32, 1-25 (2020).

7. Kassal, P., Steinberg, M. D. \& Steinberg, I. M. Wireless chemical sensors and biosensors: a review. Sens. Actuators, B Chem. 266, 228-245 (2018).

8. Lou, Z., Wang, L., Jiang, K., Wei, Z. \& Shen, G. Reviews of wearable healthcare systems: materials, devices and system integration. Mater. Sci. Eng. R. Rep. 140 100523 (2020).

9. Rao, Z. et al. Soft electronics for the skin: from health monitors to human-machine interfaces. Adv. Mater. Technol. 5, 2000233 (2020).

10. Brasier, N. \& Eckstein, J. Sweat as a source of next-generation digital biomarkers. Digit. Biomark. 3, 155-165 (2019).

11. Lu, H., Zhu, X. C., Jiang, T., Yu, J. T. \& Tan, L. Body fluid biomarkers in Alzheimer's disease. Ann. Transl. Med. 3, 1-15 (2015).

12. Broza, Y. Y. et al. Disease detection with molecular biomarkers: from chemistry of body fluids to nature-inspired chemical sensors. Chem. Rev. 119 11761-11817 (2019).

13. Choi, J., Ghaffari, R., Baker, L. B. \& Rogers, J. A. Skin-interfaced systems for sweat collection and analytics. Sci. Adv. 4, 1-10 (2018).

14. Bariya, M., Nyein, H. Y. Y. \& Javey, A. Wearable sweat sensors. Nat. Electron. 1, 160-171 (2018)

15. Kolluru, C., Williams, M., Chae, J. \& Prausnitz, M. R. Recruitment and collection of dermal interstitial fluid using a microneedle patch. Adv. Healthc. Mater. 8, 1801262 (2019)

16. Samant, P. P. \& Prausnitz, M. R. Mechanisms of sampling interstitial fluid from skin using a microneedle patch. Proc. Natl Acad. Sci. USA 115, 4583-4588 (2018).

17. Madden, J., O'Mahony, C., Thompson, M., O'Riordan, A. \& Galvin, P. Biosensing in dermal interstitial fluid using microneedle based electrochemical devices. Sens. Bio-Sens. Res. 29, 100348 (2020).

18. Lee, C. H. \& Lee, E. K. Skin-mountable flexible needle patch for minimally invasive controlled drug delivery. ONdrugDelivery 2019, 22-25 (2019).

19. Kim, Y. C., Park, J. H. \& Prausnitz, M. R. Microneedles for drug and vaccine delivery. Adv. Drug Deliv. Rev. 64, 1547-1568 (2012).

20. Larrañeta, E., McCrudden, M. T. C., Courtenay, A. J. \& Donnelly, R. F. Microneedles: a new frontier in nanomedicine delivery. Pharm. Res. 33, 1055-1073 (2016).

21. Tiffany, J. M. Tears in health and disease. Eye 17, 923-926 (2003).

22. Dartt, D. A. \& Willcox, M. D. P. Complexity of the tear film: Importance in homeostasis and dysfunction during disease. Exp. Eye Res. 117, 1-3 (2013).

23. Lam, S. M. et al. Extensive characterization of human tear fl uid collected using different techniques unravels the presence of novel lipid amphiphiles. J. Lipid Res. 55, 289-298 (2014).

24. Tomlinson, A. \& Khanal, S. Assessment of tear film dynamics: quantification approach. Ocul. Surf. 3, 81-95 (2005).

25. Kownacka, A. E. et al. Clinical evidence for use of a noninvasive biosensor for tear glucose as an alternative to painful finger-prick for diabetes management utilizing a biopolymer coating. Biomacromolecules 19, 4504-4511 (2018). 
26. Tomlinson, A., Trees, G. R. \& Occhipinti, J. R. Tear production and evaporation in the normal eye. Ophthalmic Physiol. Opt. 11, 44-47 (1991)

27. Tamhane, M., Cabrera-Ghayouri, S., Abelian, G. \& Viswanath, V. Review of biomarkers in ocular matrices: challenges and opportunities. Pharm. Res. 36, 1-35 (2019)

28. Perumal, N., Funke, S., Pfeiffer, N. \& Grus, F. H. Proteomics analysis of human tears from aqueous-deficient and evaporative dry eye patients. Sci. Rep. $\mathbf{6}$, $1-12(2016)$.

29. Kim, S., Jeon, H. J., Park, S., Lee, D. Y. \& Chung, E. Tear glucose measurement by reflectance spectrum of a nanoparticle embedded contact lens. Sci. Rep. 10 1-8 (2020).

30. Chang, R., Chen, Z., Yu, C. \& Song, J. An experimental study on stretchy and tough PDMS/fabric composites. J. Appl. Mech. Trans. ASME 86, 1-6 (2019).

31. Sim, K. Li, Y., Song, J. \& Yu, C. Biaxially stretchable ultrathin Si enabled by serpentine structures on prestrained elastomers. Adv. Mater. Technol. 4, 1-7 (2019).

32. Yin, R. et al. Soft transparent graphene contact lens electrodes for conformal full-cornea recording of electroretinogram. Nat. Commun. 9, 1-11 (2018).

33. Chimene, D., Alge, D. L. \& Gaharwar, A. K. Two-dimensional nanomaterials for biomedical applications: emerging trends and future prospects. Adv. Mater. 27, 7261-7284 (2015)

34. Kim, S. J. et al. Stretchable and transparent biointerface using cell-sheetgraphene hybrid for electrophysiology and therapy of skeletal muscle. Adv. Funct. Mater. 26, 3207-3217 (2016).

35. Kurapati, R., Kostarelos, K., Prato, M. \& Bianco, A. Biomedical uses for 2D materials beyond graphene: current advances and challenges ahead. Adv. Mater. 28, 6052-6074 (2016).

36. Xiang, Z., Liu, J. \& Lee, C. A flexible three-dimensional electrode mesh: an enabling technology for wireless brain-Computer interface prostheses. Microsyst. Nanoeng. 2, 1-8 (2016).

37. Kim, K., Kim, B. \& Lee, C. H. Printing flexible and hybrid electronics for human skin and eye-interfaced health monitoring systems. Adv. Mater. 32, 1-22 (2020).

38. Choi, S., Lee, H., Ghaffari, R., Hyeon, T. \& Kim, D. H. Recent advances in flexible and stretchable bio-electronic devices integrated with nanomaterials. Adv. Mater. 28, 4203-4218 (2016).

39. Lacour, S. P., Courtine, G. \& Guck, J. Materials and technologies for soft implantable neuroprostheses. Nat. Rev. Mater. 1, 1-14 (2016).

40. Ronkainen, N. J., Halsall, H. B. \& Heineman, W. R. Electrochemical biosensors. Chem. Soc. Rev. 39, 1747-1763 (2010)

41. Bandodkar, A. J. \& Wang, J. Non-invasive wearable electrochemical sensors: a review. Trends Biotechnol. 32, 363-371 (2014).

42. Kim, J. et al. Simultaneous monitoring of sweat and interstitial fluid using a single wearable biosensor platform. Adv. Sci. 5, 1800880 (2018).

43. Bandodkar, A. J., Jia, W. \& Wang, J. Tattoo-based wearable electrochemical devices: a review. Electroanalysis 27, 562-572 (2015).

44. Bandodkar, A. J. et al. Epidermal tattoo potentiometric sodium sensors with wireless signal transduction for continuous non-invasive sweat monitoring. Biosens. Bioelectron. 54, 603-609 (2014).

45. Guinovart, T., Bandodkar, A. J., Windmiller, J. R., Andrade, F. J. \& Wang, J. A potentiometric tattoo sensor for monitoring ammonium in sweat. Analyst 138, 7031-7038 (2013).

46. Bandodkar, A. J. et al. Tattoo-based noninvasive glucose monitoring: a proofof-concept study. Anal. Chem. 87, 394-398 (2015).

47. Kim, J. et al. Noninvasive alcohol monitoring using a wearable tattoo-based iontophoretic-biosensing system. ACS Sens. 1, 1011-1019 (2016).

48. Bakshi, P., Vora, D., Hemmady, K. \& Banga, A. K. lontophoretic skin delivery systems: Success and failures. Int. J. Pharm. 586, 119584 (2020).

49. Bae, C. W. et al. Fully stretchable capillary microfluidics-integrated nanoporous gold electrochemical sensor for wearable continuous glucose monitoring. ACS Appl. Mater. Interfaces 11, 14567-14575 (2019).

50. Reeder, J. T. et al. Waterproof, electronics-enabled, epidermal microfluidic devices for sweat collection, biomarker analysis, and thermography in aquatic settings. Sci. Adv. 5, eaau6356 (2019).

51. Koh, A. et al. A soft, wearable microfluidic device for the capture, storage, and colorimetric sensing of sweat. Sci. Transl. Med. 8, 1-14 (2016).

52. Li, S., Ma, Z., Cao, Z., Pan, L. \& Shi, Y. Advanced wearable microfluidic sensors for healthcare monitoring. Small 16, 1-15 (2020).

53. Martín, A. et al. Epidermal Microfluidic electrochemical detection system: enhanced sweat sampling and metabolite detection. ACS Sens. 2, 1860-1868 (2017).
54. Nyein, H. Y. Y. et al. Regional and correlative sweat analysis using highthroughput microfluidic sensing patches toward decoding sweat. Sci. Adv. 5, eaaw9906 (2019).

55. Nyein, H. Y. Y. et al. A wearable microfluidic sensing patch for dynamic sweat secretion analysis. ACS Sens. 3, 944-952 (2018).

56. Yang, $Y$. et al. A laser-engraved wearable sensor for sensitive detection of uric acid and tyrosine in sweat. Nat. Biotechnol. 38, 217-224 (2020).

57. Diabetes, D. O. F. Diagnosis and classification of diabetes mellitus. Diabetes Care 32, S62-S67 (2009).

58. Lee, H. et al. A graphene-based electrochemical device with thermoresponsive microneedles for diabetes monitoring and therapy. Nat. Nanotechnol. 11 566-572 (2016).

59. Lee, $H$. et al. Wearable/disposable sweat-based glucose monitoring device with multistage transdermal drug delivery module. Sci. Adv. 3, 1-9 (2017).

60. Jang, K. I. et al. Self-assembled three dimensional network designs for soft electronics. Nat. Commun. 8, 1-10 (2017).

61. Xu, S. et al. Assembly of micro/nanomaterials into complex, three-dimensional architectures by compressive buckling. Science 347, 154-159 (2015).

62. Jang, K. I. et al. Soft network composite materials with deterministic and bioinspired designs. Nat. Commun. 6, 1-11 (2015).

63. Li, J., Liang, J. Y., Laken, S. J., Langer, R. \& Traverso, G. Clinical opportunities for continuous biosensing and closed-loop therapies. Trends Chem. 2, 319-340 (2020).

64. Ribet, F., Stemme, G. \& Roxhed, N. Real-time intradermal continuous glucose monitoring using a minimally invasive microneedle-based system. Biomed. Microdevices 20, 101 (2018).

65. Chang, H. et al. A swellable microneedle patch to rapidly extract skin interstitial fluid for timely metabolic analysis. Adv. Mater. 29, 1-8 (2017).

66. Miller, P. R. et al. Extraction and biomolecular analysis of dermal interstitial fluid collected with hollow microneedles. Commun. Biol. 1, 1-11 (2018).

67. Goud, K. Y. et al. Wearable electrochemical microneedle sensor for continuous monitoring of levodopa: toward Parkinson management. ACS Sens. 4 2196-2204 (2019).

68. Mohan, A. M. V., Windmiller, J. R., Mishra, R. K. \& Wang, J. Continuous minimallyinvasive alcohol monitoring using microneedle sensor arrays. Biosens. Bioelectron. 91, 574-579 (2017)

69. Chen, Y. et al. Skin-like biosensor system via electrochemical channels for noninvasive blood glucose monitoring. Sci. Adv. 3, 1-8 (2017).

70. Dieckmann, G., Goyal, S. \& Hamrah, P. Neuropathic corneal pain: approaches for management. Ophthalmology 124, S34-S47 (2017).

71. Kim, J., Cha, E. \& Park, J. U. Recent advances in smart contact lenses. Adv. Mater Technol. 5, 1900728 (2020).

72. Farandos, N. M., Yetisen, A. K., Monteiro, M. J., Lowe, C. R. \& Yun, S. H. Contact lens sensors in ocular diagnostics. Adv. Healthc. Mater. 4, 792-810 (2015).

73. Tseng, R. C., Chen, C. C., Hsu, S. M. \& Chuang, H. S. Contact-lens biosensors. Sensors (Switzerland) 18, 2651 (2018).

74. Thomas, N., Lähdesmäki, I. \& Parviz, B. A. A contact lens with an integrated lactate sensor. Sensors Actuators. B Chem. 162, 128-134 (2012).

75. Yao, H., Shum, A. J., Cowan, M., Lähdesmäki, I. \& Parviz, B. A. A contact lens with embedded sensor for monitoring tear glucose level. Biosens. Bioelectron. 26 3290-3296 (2011)

76. Keum, D. H. et al. Wireless smart contact lens for diabetic diagnosis and therapy. Sci. Adv. 6, eaba3252 (2020).

77. Lee, M. S. et al. High-performance, transparent, and stretchable electrodes using graphene-metal nanowire hybrid structures. Nano Lett. 13, 2814-2821 (2013).

78. Kim, J. et al. Wearable smart sensor systems integrated on soft contact lenses for wireless ocular diagnostics. Nat. Commun. 8, 1-8 (2017).

79. Park, J. et al. Soft, smart contact lenses with integrations of wireless circuits, glucose sensors, and displays. Sci. Adv. 4, eaap9841 (2018).

80. $\mathrm{Ku}, \mathrm{M}$. et al. Smart, soft contact lens for wireless immunosensing of cortisol. Sci. Adv. 6, 1-9 (2020)

81. Alipour, F., Khaheshi, S., Soleimanzadeh, M., Heidarzadeh, S. \& Heydarzadeh, S. Contact lens-related complications: a review. J. Ophthalmic Vis. Res. 12 193-204 (2017)

82. Jeerapan, I. \& Poorahong, S. Review — flexible and stretchable electrochemical sensing systems: materials, energy sources, and integrations. J. Electrochem Soc. 167, 037573 (2020).

83. Song, Y. et al. Wireless battery-free wearable sweat sensor powered by human motion. Sci. Adv. 6, 1-11 (2020).

84. LV, J. et al. Sweat-based wearable energy harvesting-storage hybrid textile devices. Energy Environ. Sci. 11, 3431-3442 (2018). 
85. Bandodkar, A. J. et al. Soft, stretchable, high power density electronic skinbased biofuel cells for scavenging energy from human sweat. Energy Environ. Sci. 10, 1581-1589 (2017).

86. $\mathrm{Yu}, \mathrm{Y}$. et al. Biofuel-powered soft electronic skin with multiplexed and wireless sensing for human-machine interfaces. Sci. Robot. 5, 1-14 (2020).

87. Jeerapan, I., Sempionatto, J. R. \& Wang, J. On-body bioelectronics: wearable biofuel cells for bioenergy harvesting and self-powered biosensing. Adv. Funct. Mater. 30, 1-18 (2020).
88. Bandodkar, A. J. et al. Battery-free, skin-interfaced microfluidic/electronic systems for simultaneous electrochemical, colorimetric, and volumetric analysis of sweat. Sci. Adv. 5, 1-16 (2019).

89. Ciui, B. et al. Wearable wireless tyrosinase bandage and microneedle sensors: toward melanoma screening. Adv. Healthc. Mater. 7, 1-9 (2018).

90. Yao, $\mathrm{H}$. et al. A contact lens with integrated telecommunication circuit and sensors for wireless and continuous tear glucose monitoring. J. Micromechanics Microengineering 22, 075007 (2012). 